\title{
GEOINFORMATION SYSTEMS AS A TOOL OF THE INTEGRATED TOURIST SPACES MANAGEMENT
}

\author{
VICTOR KOLESNIKOVICH ${ }^{1}$, YULIA BYCHENKOVA ${ }^{2}$, ALEKSANDR VERNIGOR $^{3}$ \\ ${ }^{1}$ Belarusian State University of Physical Culture, Institute of Tourism \\ ${ }^{2}$ Moscow State University of Tourism and Service, Smolensk Branch \\ ${ }^{3}$ Moscow State University of Tourism and Service, Department of Service
}

\author{
Mailing address: Victor Kolesnikovich, Belarusian State University of Physical Culture, Institute of Tourism, \\ 105/434 Peramozhcau Ave., Minsk, tel.: +375 447 772737, fax: +375 172508008 , \\ e-mail: ums_minsk@tut.by
}

\begin{abstract}
Introduction. Currently tourist activity management is in need of creating special conditions for the development of integrated management tools based on the general information and analytical base. Material and methods. The creation of architecture and the content of geoinformation and hybrid information systems are oriented at the usage of the Integrated Tourist Spaces Management (ITSM) to set up a specific claim related to the features of management model. The authors created the concept of tourist space. The information and the analytical system are used to create the information model of tourist space. Information support development of ITSM system is a sort of a hybrid system: an expert system constructed on the basis of GIS. Results and conclusions. By means of GIS collecting, storage, analysis and graphic visualization of spatial data and the related information on the objects presented in an expert system is provided. The offered approach leads to the formation of an information system and the analytical maintenance of not only human decision-making, but it also promotes the creation of new tourist products based on more and more differentiated inquiries of clients or a ratio of the price and quality (from the point of view of satisfaction of inquiries).
\end{abstract}

Key words: geographical information system (GIS), hybrid information system, tourist space, tourist potential, recreational resources, spatial temporary data, closing expenses

\section{Introduction}

Tourist activity is an important social and economic component of economic systems of many states. Due to the geographical position and the transport infrastructure, the Republic of Belarus and adjacent territories of the Russian Federation possess considerable tourist potential. The improvement of quality of recreational resources management is a problem of increasing tourist potential [1] realized by means of:

» diversification of tourist activity due to a fuller and effective use of recreational resources;

» development of warning measures for ensuring safety in terms of the quality of recreational resources;

» infrastructure improvements;

» human development.

The inclusion of a special component into a regional strategy and programs of social and economic development has to become the mechanism of implementing new approaches to the development of tourist activity. It is necessary to introduce innovations and to achieve synergy by means of the coordinated development of tourist activity and its complex information and analytical support.

Currently tourist activity management is generally implemented on the basis of the branch principle by the agencies that report to various ministries and departments. It confirms the need of creating conditions for the development of instruments of integrated management based on the general information and analytical base.

\section{Material and methods}

The information used needs spatial and territorial binding. Information support development of the ITSM system is a sort of a hybrid system: an expert system constructed on the basis of GIS.

By means of GIS collecting, storage, analysis and graphic visualization of spatial data and the related information on the objects presented in an expert system is provided. GIS contains data on real objects in a digital form. It includes the set of functional implementing operations of geoinformation technologies.

Within an expert system all operations are supported by a standard and legal program, and hardware. Information, personnel and organizational support is also provided.

The article [2] introduces the concept of spatial data as figures regarding the objects including data on their location and properties (spatial and non-spatial attributes).

The change of spatial data over time means introducing a temporary parameter for the description and simulation of the processes involved. The details of these processes are registered as spatial temporary data $[2,3]$.

Temporary characteristics are represented in the form of data acquisition dates:

» define life cycle,

» define location change,

» define changes of spatial objects properties in time.

For the geoinformation representation the spatial world surrounding us is defined [3] by means of basic concepts: essence, 
properties, communications.

In the analysis of spatial temporary processes we can distinguish two types of the entity:

" the discrete entity - objects of supervision presented to GIS for which vector formats of data are used;

" the continuous varieties represented by a net or raster formats.

Properties represent concepts which characterize an entity.

Communications represent concepts which describe the relations between:

$»$ entities,

» properties,

» entities and properties.

The elements of reality simulated in the data base of GIS have two images: real object $\leftrightarrow$ simulated object (database object).

Real object - an interesting phenomenon of the world, which cannot be divided into the phenomena of the same type. Simulated object is a digital representation of all real objects or some parts of them. The method of the digital representation of the phenomenon depends on the basic scale and some other factors. Each type of a real object represents certain database objects. Database objects can be grouped into the layers, which are called overlays, coverings and subjects. One layer can represent a single type of objects or groups of conceptually connected types. The database includes:

» digital versions of real objects, e.g. buildings;

» digital versions of properties of the card marked out artificially, e.g. borders;

» artificial objects made specifically for creating a DB, e.g. pixels. tasks:

Analytical GIS are developed in order to solve the following

» estimating and interpreting communications between properties;

» predicting, detecting and recognising targeted (previously unknown) properties of supervised objects;

» forecasting the development of spatial temporary processes.

\section{Results}

The offered approach allows to construct an information model and to use it for the representation of tourist space. Let us call: object of supervision - point $O=O(x, y, h)$, unambiguously defined by a geographical position, where $x$ - width, $y$ - longitude, $h$ - height [2]; supervision area-area $\mathrm{O}^{N}=\left\{\mathrm{O}_{1}, \ldots, \mathrm{O}_{N}\right\}, N \in \mathrm{N}$, consisting of $N$ objects of supervision. As an object of a DB the object of supervision is considered $O=O(x, y, h)$. Figure 1 illustrates the representation of the supervised object $O=O(x, y, h)$ in the GIS.

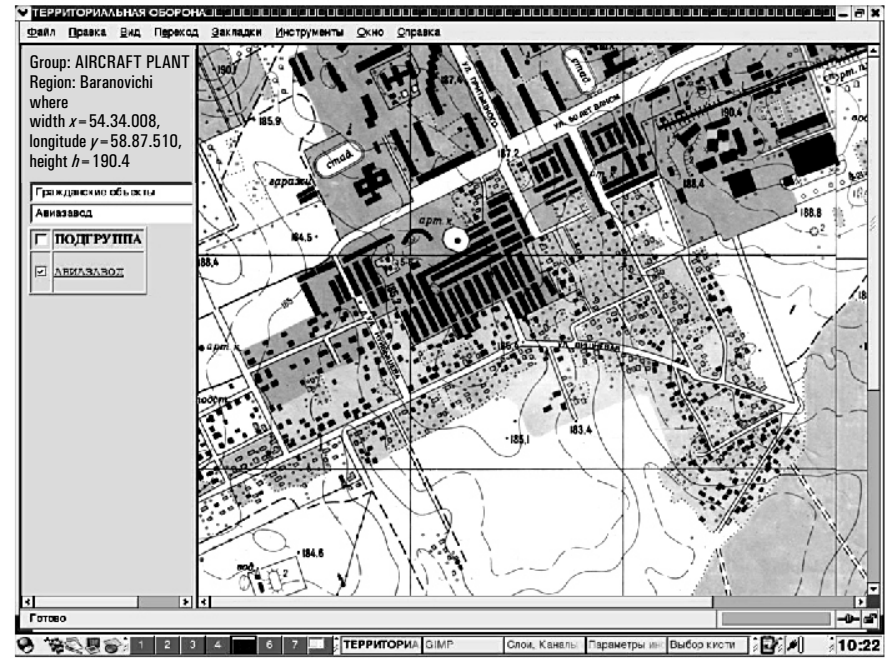

Figure 1. Representation of a supervised object within GIS is illustrated by $O=O(x, y, h)$ (the object is designated by a square of two colors)

The following vector of states characterizes each object of supervision $O=O(x, y, h), S^{O}(t)=\left\{S_{1}^{O}(t), \ldots S_{q}^{O}(t)\right\}$, where $S_{k}^{O}(t)$, $k=\overline{1, q}, q \in N$ - state of $k$ - st property object of supervision $O=O(x, y, h)$ in a time point $t, S^{O}(t)-q$ - dimensional process with discrete $(t \in Z)$ or continuous $(t \in R)$ time. Different objects can be characterized by various quantities $q \in \mathrm{N}$ and a set of properties $S_{k}^{O}(t)$.

Figure 2 illustrates the representation of attributes for the same supervised object $O=O(x, y, h)$ within the GIS. The quantity and set of properties of a supervised object are defined by users of the created GIS.

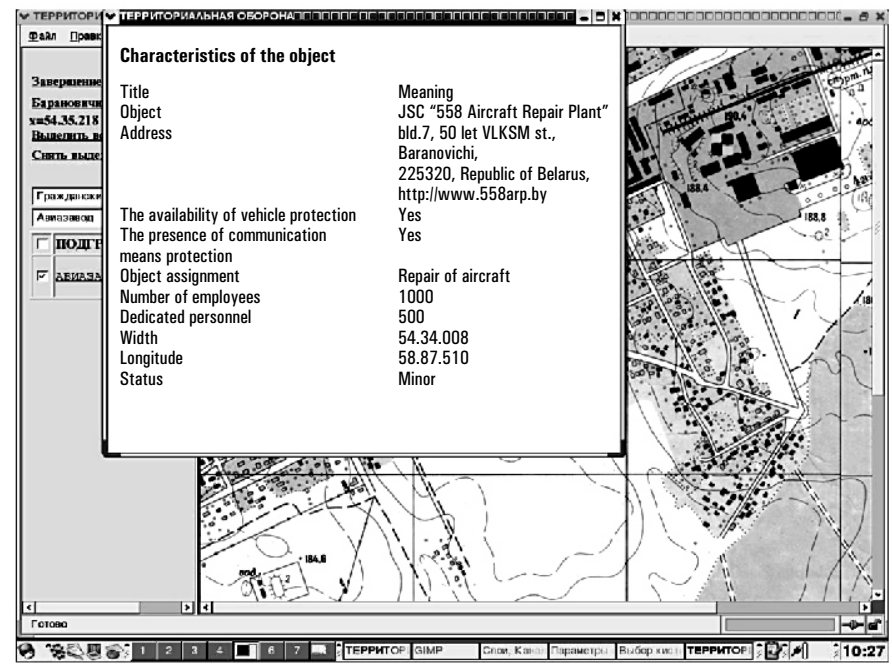

Figure 2. Representation of attributes of the same supervised object within GIS is illustrated by $O=O(x, y, h)$ - the object

is designated by a square of two colors. The information and analytical bases are in Russian (corresponding to attributes of objects) 
Vector of states $S^{O}(t)$ of the object of supervision $O=O(x, y, h)$ for any time point submits statistical data. These statistical data are used for calculating integrated indicators necessary for the analysis of recreational resources [1,2]. Various objects of supervision can be integrated on a territorial, branch and categorical sign in the field of supervision $\mathrm{O}^{N}=\left\{\mathrm{O}_{1}, \ldots, \mathrm{O}_{N}\right\}, N \in \mathrm{N}$.

Informative representation of the object allows to realize the information model of tourist space. In the work of Dzhandzhugazova the formalized category "tourist space" was coined and a technique of studying tourist territories relying on the category "tourist space" was created [4]. Tourist space is "a special type of the space that exists as a logical form (structure) and it consists of elements of the different nature united by the uniform purpose - satisfaction of the needs of people concerning leisure, rest and recreation".

For an economic assessment of natural recreational resources we use costs of "production" of recreation - closing expenses. The development of an economic-mathematical model is based on observing the following requirements:

» as a criterion function, the criterion of a minimum of the current and one-time costs of the set output during the creation and designed project functioning is applied;

» models have to provide dual estimates in the most convenient way for the analysis and the subsequent use in economic calculations;

» a degree of an aggregation of initial indicators into the model has to provide a certain stability of received indicators of optimum estimates in relation to a possible variation of basic data.

Within implementation of the project information resourcetourist interactive the Live Card cards are created [5]. The implementation of the Live Russia Card project is an example of creating the described information and an analytical system. The representation of objects is illustrated in the drawing.

\section{Discussion}

The implementation of the project requires creating a technique of studying tourist spaces and defining standards of classification, the description and a graphic representation of various objects of display (tourist resources) (fig. 3).

The algorithm described below is a developed result:

1. Allocation of territorial objects with the use of the administrative card. Grouping and fixing territorial objects to certain researchers.

2. Allocation of key signs of various types of tourist space and definition of the list of objects in the system of a certain type of tourist space:

» natural space,

» cultural and historical space,

» recreational space,

» service space,

» anthropological space,

» event space.

3. Formation of "a kernel of signs" characterizing the allocated objects within separate types of tourist space and development of forms for the characteristics (description) of the object.

4. Formation of the system of processing, systematization and standardization of received information for the purpose of its placement on an information portal.

5. Creation of the effective system of "feedback" on an information portal which will store information on tourist territories in a section of the allocated types of tourist space.
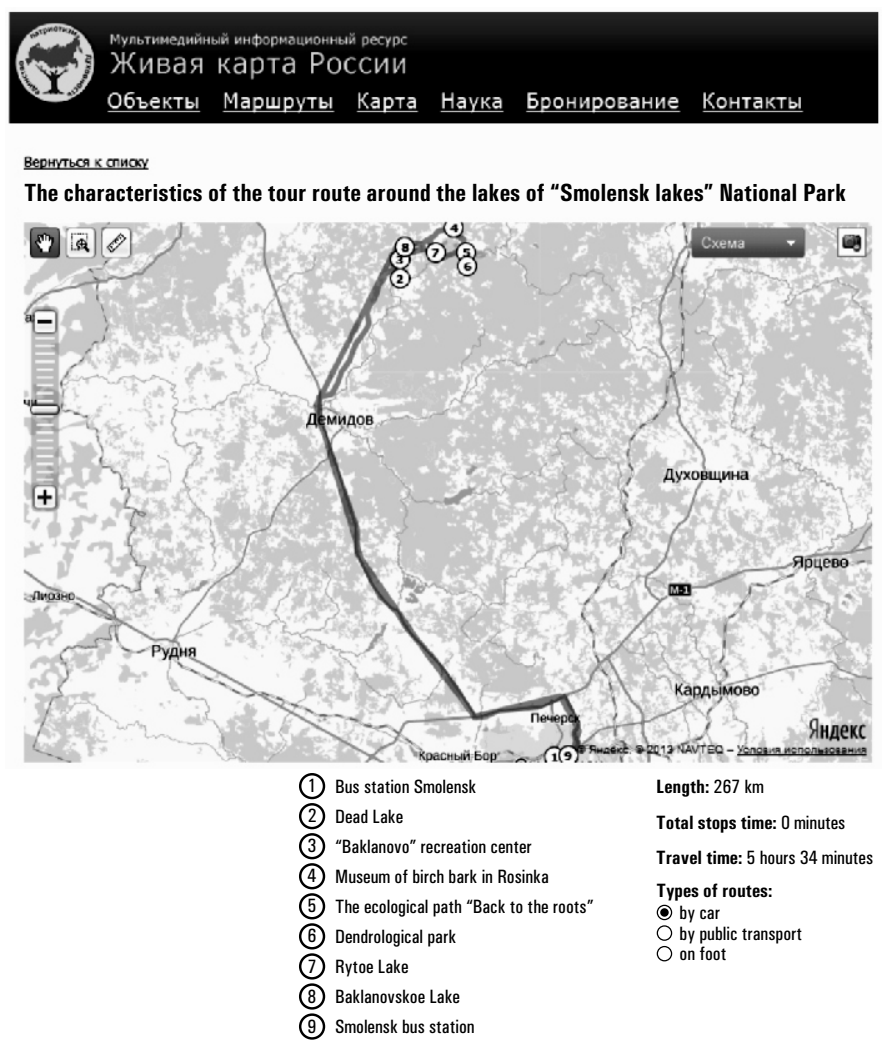

Characteristics

Target: Tourists of a "Pearl of the Smolensk lake area" - lakes, their rich flora and fauna, historical and cultural sights of the Park.

Target group: A specific product can attract a wide range of customers because of its cognition and low cost: school groups, youth, families with children, persons interested in environmental tourism. Types of routes: Combined (bus, bike, on foot)

Route duration: 2 days

Nutrition and sanitation needs: "Baklanovo" recreation center

Shoes, clothes, behavior rules on the route: Convenient, not hampering and closed clothing and footwear.

Figure 3. A graphic representation of various objects of display (tourist resources)

A technique offered by Dzhandzhugazovoy is realized in the form of the information portal filled with the use of opportunities of crowdsourcing. The realized approach differs from a large number of existing tourist portals:

» the uniform classification of tourist objects used for all territories,

» a possibility of combining various information layers,

» implementing uniform management of an information portal,

» using technologies of crowdsourcing for content formation.

\section{Conclusions}

The use of the obtained results as a basis for creating the administrative decisions adoption support instrument requires its expansion to a hybrid information system.

It is necessary to create thematic layers with various levels of the access, information filled with a wider range allowing to make an assessment of economic efficiency of using tourist space and to reveal reserves on the basis of creating multiplefactor models. 
The offered approach leads to the formation of an information system and the analytical maintenance of not only human decision-making, but it also promotes the creation of new tourist products based on more and more differentiated inquiries of clients or a ratio of the price and quality (from the point of view of satisfaction of inquiries).

\section{Literature}

1. Kolesnikovich V.P. (2008). Recreational zones of the Belarusian Polesye - the most important factor of development of tourism. Messenger of the Moscow State Regional University Series Natural Sciences 123(1), 75-81. [in Russian]
2. Sanjuk (Lapitskaja) N.V. (2005). GIS use in the analysis of existential data. News of the Belarusian Engineering Academy 1(19)/2, 93-97. [in Russian]

3. Gitis V.G., Ermakov B.V. (2004). Bases of existential forecasting in geoinformatics. Moscow: Fizmatlit. [in Russian]

4. Dzhandzhugazova E. A. (2010). Tourism and innovative development: project 'Live Russia Map' of Moscow State University of Tourism and Service. Modern Problems of Service and Tourism 3, 66-72. [in Russian]

5. http://www.livemaprus.ru

Submitted: February 13, 2014

Accepted: May 15, 2014 